\title{
CHANGE OF CALIBRATION PARAMETERS IN FREQUENTLY USED VIALS IN BENZENE SCINTILLATION COUNTING
}

\author{
HERBERT HAAS \\ Desert Research Institute, Water Resources Center, P.O. Box 19040, Las Vegas, Nevada \\ 89132-0040 USA
}

\begin{abstract}
Scintillation counting vials used for counting synthesized benzene accumulate an inside coating which is insoluble in benzene. This coating reduces the photon transfer from the vial and lowers its observed background. Age calculations that include calibration data collected with other vials may be inaccurate if this effect is not considered. Frequent cleaning of the counting vials with strong acid solutions keeps the coating from accumulating.
\end{abstract}

A sample counting vial cleaned only with photospectrometric grade benzene between 67 different sample benzene fillings made in a 20 -month period showed, during counting, an efficiency lowered by $2.8 \%$ and a background lowered by $8.7 \%$. Age calculations for a $10 \mathrm{ka}$ old sample made with calibration data from "matched" vials would yield a result too old by 515 yr.

\section{INTRODUCTION}

The accurate calibration of scintillation counters requires long-term observation of several variables. Among these most commonly measured parameters are: 1) background; 2) counter efficiency; 3) quenching; and 4) individual vial characteristics.

I propose another variable parameter mostly affecting laboratories involved in high-precision counting: inside cleanliness of counting vials. I have observed that benzene synthesized from acetylene with a vanadium oxide catalyst causes a faint and largely invisible coating to build up on the inside wall of counting vials. A study in progress indicates several potential sources for impurities in the benzene: volatile compounds of stopcock grease; elastomers in O-rings; oil vapors from vacuum pumps; impurities in the acetylene; and byproducts from the catalytic conversion of acetylene. The vial coating is not soluble in benzene or acetone, reducing the optical efficiency of the vial. Restoration of the original counting performance requires a thorough cleaning of the vial with an acidic solution.

One of two different methods of counter calibration is used by most radiocarbon laboratories:

1. A selected group of vials with nearly identical counting characteristics is divided into calibration vials and sample counting vials. The calibration vials are filled with background or modern standards and are sealed permanently. The sample counting vials are filled repeatedly with freshly synthesized benzene cocktail solutions. Standard measurements are not performed on a routine basis using the sample vials.

2. No attempt is made to match vials. Each counting vial is calibrated on a planned schedule with background and modern standard solutions, interspersed between sample counts. Vials are not sealed permanently and none is designated solely for calibration measurements.

Any gradual change of vial characteristics will affect the sample counting results of the two methods in different ways. In the first method, changes in the count rate are not detected and will introduce uncertainties in the calculation of ${ }^{14} \mathrm{C}$ results. In the second, the extent of sample counting rate reduction is offset by equivalent changes in the efficiency of detecting decays in calibration cocktails. 


\section{METHODS}

\section{Strategy}

The research presented here is entirely based on measurements made with calibration cocktails. These include benzene synthesized from anthracite and from the oxalic acid standard. Cocktails derived from anthracite are used for background determinations and the counting results are presented as total counts per minute. Modern Standard measurements are made with cocktails derived from either of the two oxalic acid standards, HOx I or HOxII. These counting results are normalized to $1 \mathrm{~g}$ carbon and are adjusted by factors of 0.95 or 0.73685 , respectively, which are assigned to the two oxalic acid versions. All measurements presented in this paper are part of the routine operation of the counter. Sample cocktails were counted between calibration measurements. All cocktails are made from recently synthesized benzene to which 0.91 weight-percent butyl-PBD scintillator is added in powder form. Calibration cocktails are used repeatedly in all counting vials. It is therefore important to note that the vials were exposed over the period of observation to $c a$. forty fillings of fresh cocktails.

\section{Techniques}

A square quartz counting vial, commercially obtainable as a spectrophotometric cell (Haas 1979), was monitored for 20 months. This vial was used in rotation with other vials in the LKB Rackbeta $1217^{\mathrm{TM}}$ counter. Sample or calibration cocktails were filled into the vial and counted for $c a .2$ days. The 3-cc volume of the cocktail filling was kept constant for all fillings within $\pm 5 \mathrm{mg}$. The cocktails were then returned to storage containers and the counting vial was cleaned with high-purity benzene. All background and modern standard measurements were plotted and a decline of $2.8 \%$ efficiency was observed for the modern standard measurements over the period. A rigorous cleaning returned the efficiency of the vial to the initial value. The final background count rate value was likewise depressed by $8.7 \%$ compared to that recorded after cleaning. A second vial was monitored for three months. It showed a similar pattern, with a decline in modern count rate efficiency of $1.5 \%$ and a background reduction of $3.6 \%$.

Figure 1A shows these effects for a modern standard (prepared from oxalic acid) and Figure 1B for a background cocktail. The vial was cleaned previously several months before the beginning of the plotted time period. Figure $1 \mathrm{~A}$ shows how the cleaning of the vial returned its counting efficiency to the value observed during its initial uses.

No chemical analysis was made to identify the substance that had accumulated on the inside walls of the quartz vials. One can readily observe the coating, however, by a difference in the wetting property of the inside surface. A liquid introduced into the vial (benzene, acetone or water) will stay as a thick fluid film up to the height to which the vial is ordinarily filled. No film forms on the wall surface above the filling level, and the liquid runs off that surface quickly, often forming small droplets. This can be seen more clearly by adding a small amount of metylene blue to a water and acetone mixture. In frequently used vials, the coating accumulates more rapidly and becomes visible in the dry vial after a few months as a very faint grayish stain.

The origin of the substance that coats the vials is unknown. Coleman (1973) suggested the formation of a catalytic byproduct of high molecular weight. Traces of stopcock grease from the vacuum system might be another explanation. It is a possible source in our systems where a few grease-sealed components are used. 


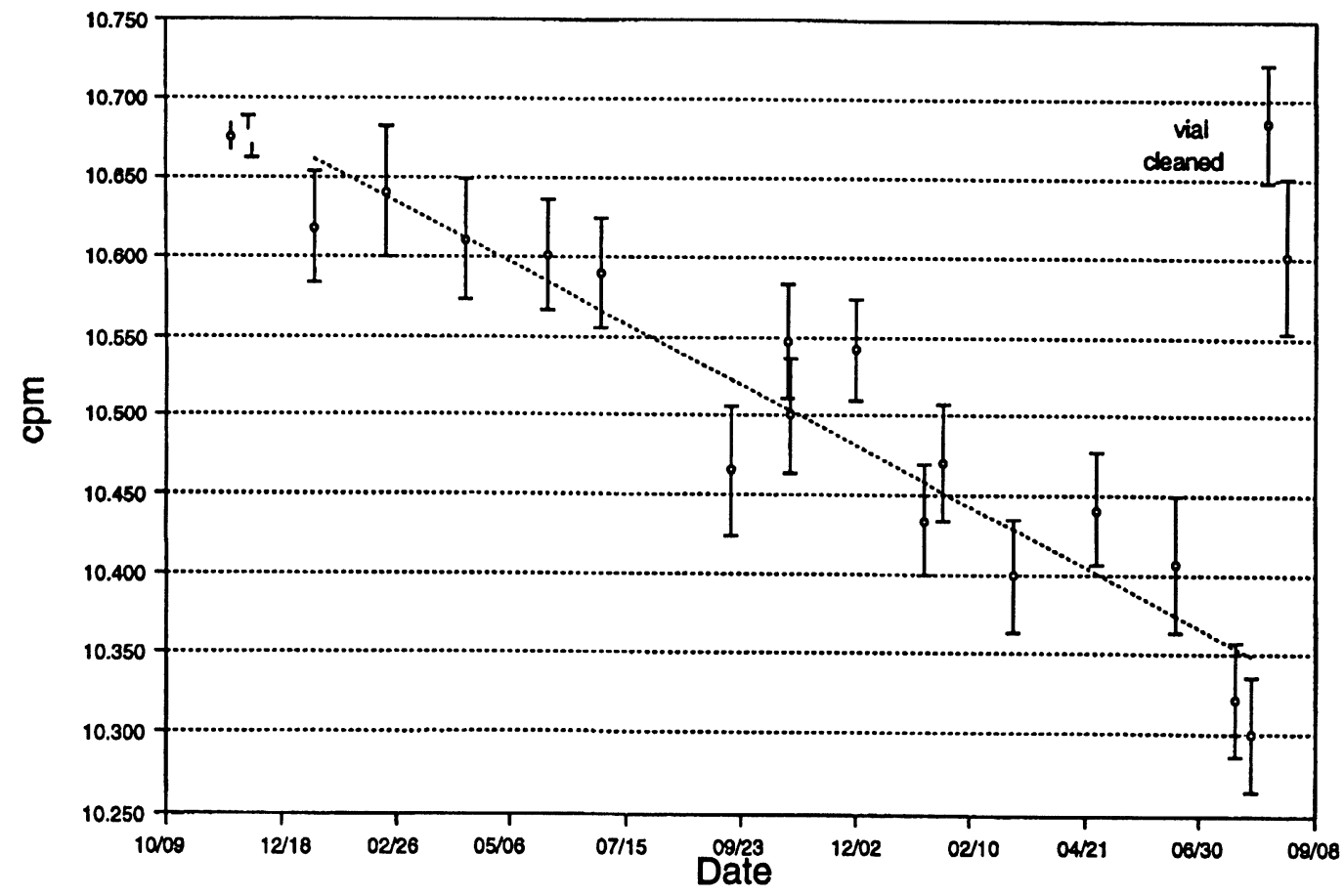

Fig. 1A. Regression showing the decline in vial efficiency and the restored efficiency after cleaning (Vial 41, oxalic acid).

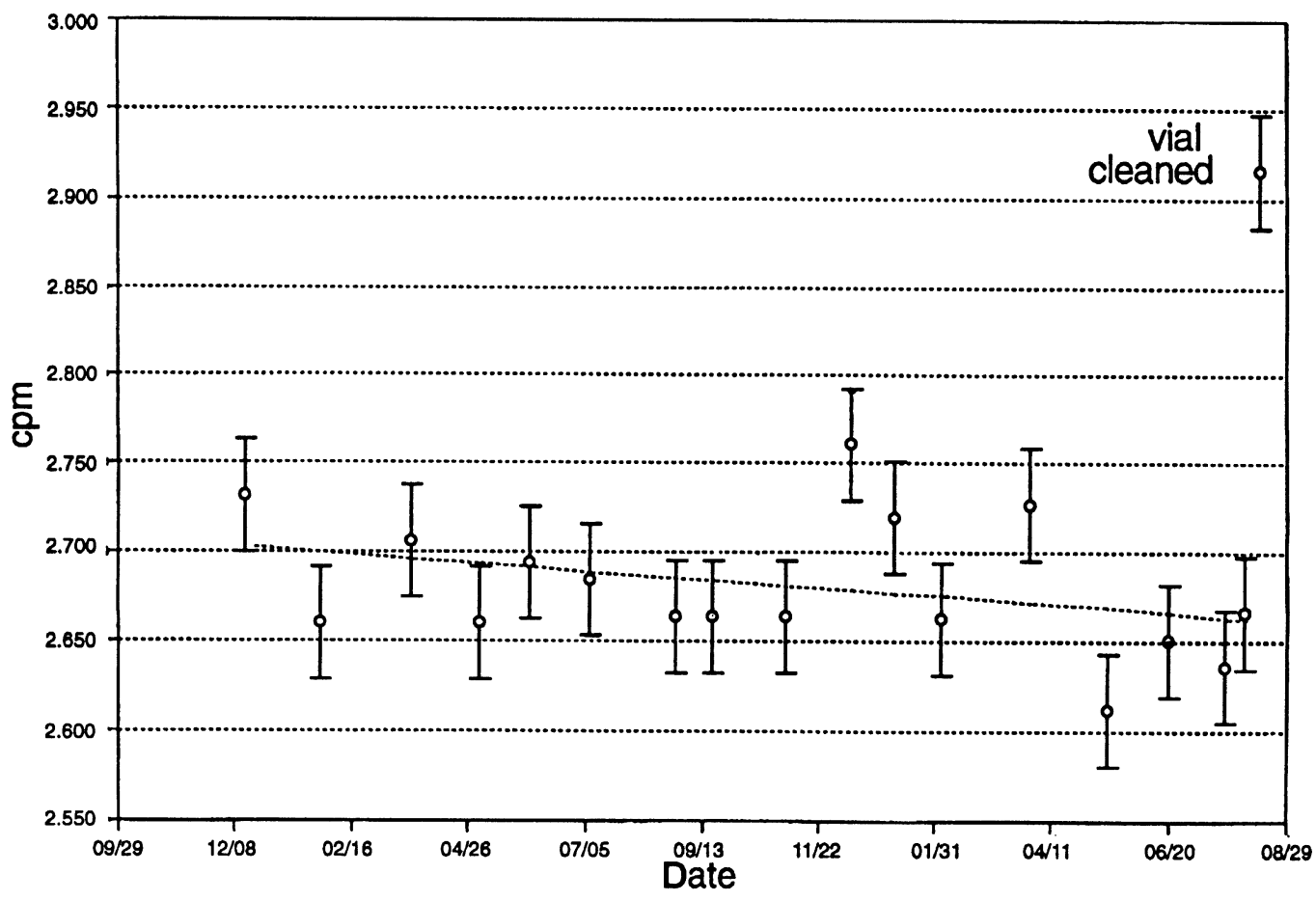

Fig 1B. Regression showing the decline in vial background and the rise in background after cleaning (Vial 41, background). 
The vials were cleaned as follows:

Submerge the vial for 12-24 $\mathrm{h}$ in concentrated sulfuric, nitric or chromic acid. I use the commercially available substitute for chromic acid "Nochromix". After thorough water rinses, the vial is boiled in high-purity distilled water, then dried. Rinses in spectrophotometric-grade benzene conclude the procedure.

\section{Measurements}

Linear regressions performed on the data (Fig. 1A, B) provide the following data for a $10 \mathrm{~mm}^{2}$ (inside dimension) quartz vial of $5 \mu \mathrm{l}$ capacity over a period of 20 months:

Background of the cleaned vial

Reduction of background in coated vial

Modern standard count rate, per gram C

Reduction of modern standard count rate

$$
\begin{aligned}
& 2.916 \mathrm{cpm} \\
& 0.254 \mathrm{cpm} \\
& 10.644 \mathrm{cpm} \\
& 0.295 \mathrm{cpm} .
\end{aligned}
$$

The cause of the count rate reduction was investigated further by plotting the decay energy spectra of a modern standard. The standard was first counted in the coated vial and then in the same vial after cleaning. Figure 2 shows the shift to lower energy of the curve from the coated vial. The observed effect is similar to quenching. The reduction of the count rate of an active sample can therefore be explained with the shift of the energy spectrum relative to a fixed energy window. Background counts register in a much broader spectrum without a distinct maximum. The reduction in count rate is not explained therefore by a pulse energy loss alone.

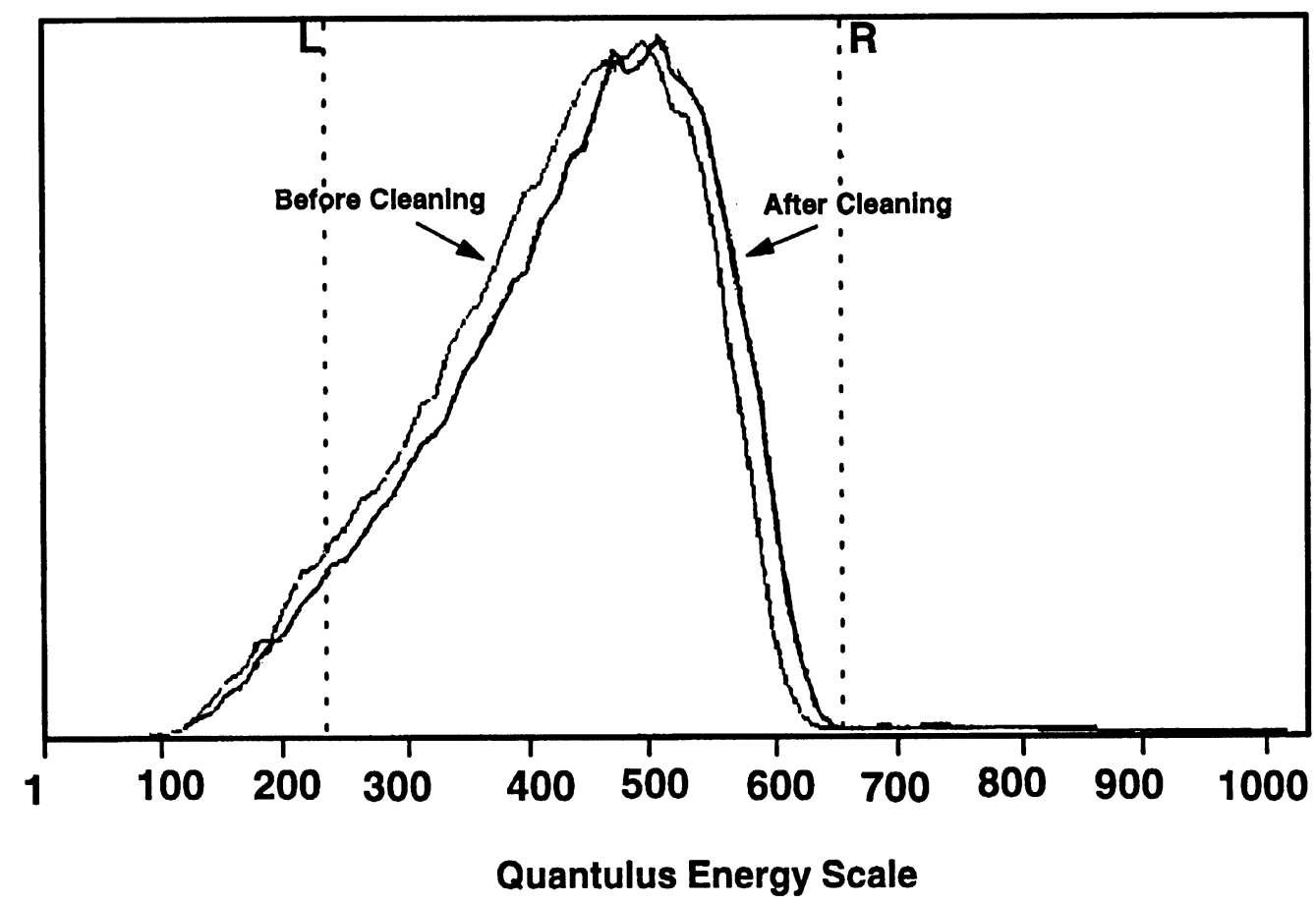

Fig. 2. Shift in energy spectrum due to vial contamination. Left spectrum measured in coated vial, right spectrum in clean vial. 


\section{RESULTS}

The observed changes of calibration values (modern standard and background) from two vials were used to calculate and plot potential uncertainties in sample ages measured with a "coated" vial. A reversed age calculation was used to compute a net sample count rate for a clean vial. A range of assumed ${ }^{14} \mathrm{C}$ ages was used in the tabulation. The rate was then reduced by the percentage of the observed count-rate decline of the modern standard in the coated vial. To this computed count rate was added the background in the coated vial. The result is the expected raw count rate in the coated vial. Finally, an age calculation was performed with the computed raw count rate, whereby the background and modern standard rates of a clean vial were used. Figure 3 shows the differences of the assumed and computed ages. The four curves illustrate different time periods of coating accumulation. An accumulation linear with time is assumed.

From Figure 3, one can calculate that a vial cleaned in benzene for 10 months could eventually yield an uncertainty of $+160 \mathrm{yr}$ for a real sample age of $2 \mathrm{ka}$. This magnitude of uncertainty assumes that modern and background count rates were based on separate, dedicated standard vials that are not exposed to the accumulating coating. Laboratories that practice quasi-simultaneous counting are exposed to such an uncertainty.

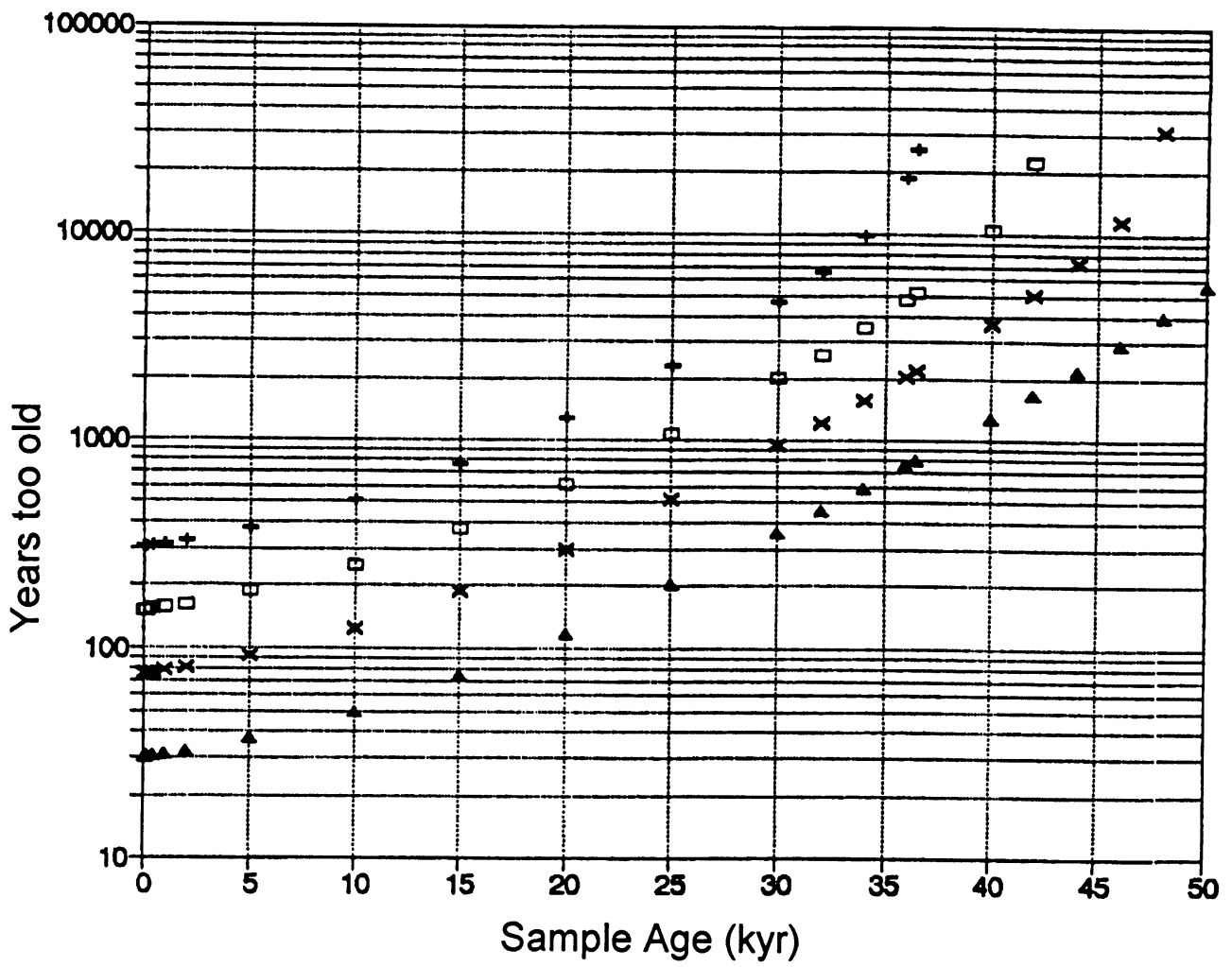

Fig. 3. Uncertainty in calculated ages due to presence of a contaminant on the vial wall

$+=$ maximum accumulation of coating over 20 months; total observed efficiency and background changes

$\square=$ accumulation over 10 months; $50 \%$ of the above changes

$X=$ accumulation over 5 months; $25 \%$ of the above changes

$\Delta$ = accumulation over 2 months; $10 \%$ of the above changes 


\section{CONCLUSION}

The introduction of a contaminant into the counting vials by synthesized benzene is likely to depend in its magnitude on the design of the benzene synthesis system and on the materials used in the vacuum lines. Most laboratories tend to be exposed to this problem. Experimentation with vial cleaning methods and direct observation of counting efficiency in all vials with standard solutions is recommended for high-precision counting systems. Frequent thorough cleaning of counting vials, using an acidic cleaning agent (Nochromix) is required if calibration parameters are established with dedicated calibration vials only. A calibration method in which the calibration parameters are directly and continuously established with each vial removes the risk of undetected discrepancies between assumed and real data. It allows a less rigorous schedule of vial cleaning.

\section{ACKNOWLEDGMENTS}

Research shown in this paper was carried out at the laboratory's locations at Southern Methodist University and at the Desert Research Institute. The laboratory is supported by NSF Core Grant No. BNS 9111714 . The manuscript was prepared for publication by Rebecca Gardiner; Judy Marchland helped with the graphic plots.

\section{REFERENCES}

Coleman, D. D., Liu, C. L., Dickerson, D. R. and Frost, R. R. 1973 Improvement in trimerization of acetylene to benzene for radiocarbon dating with a commercially available vanadium oxide catalyst (Abstract). In Rafter, T. A. and Grant-Taylor, T., eds., Proceedings of the 8 th International Conference on ${ }^{14} \mathrm{C}$ Dating. Wellington,
New Zealand, Royal Society of New Zealand: 158. Haas, H. 1979 Specific problems with liquid scintillation counting of small benzene volumes and background count rate estimation. In Berger, R. and Suess, H. E., eds., Radiocarbon Dating. Berkeley, University of California Press: 246-255. 